This item was submitted to Loughborough's Research Repository by the author.

Items in Figshare are protected by copyright, with all rights reserved, unless otherwise indicated.

\title{
Probabilistic approach to nonlinear wave-particle resonant interaction
}

PLEASE CITE THE PUBLISHED VERSION

https://doi.org/10.1103/PhysRevE.95.023204

\section{PUBLISHER}

(C) American Physical Society

VERSION

AM (Accepted Manuscript)

\section{PUBLISHER STATEMENT}

This work is made available according to the conditions of the Creative Commons Attribution-NonCommercialNoDerivatives 4.0 International (CC BY-NC-ND 4.0) licence. Full details of this licence are available at: https://creativecommons.org/licenses/by-nc-nd/4.0/

\section{LICENCE}

CC BY-NC-ND 4.0

\section{REPOSITORY RECORD}

Artemyev, A.V., Anatoly Neishtadt, Alexei Vasiliev, and D. Mourenas. 2019. "Probabilistic Approach to Nonlinear Wave-particle Resonant Interaction”. figshare. https://hdl.handle.net/2134/24397. 


\title{
Probabilistic approach to nonlinear wave-particle resonant interaction
}

\author{
Artemyev A. V. ${ }^{1,2}$, Neishtadt A. I. ${ }^{3,2}$, Vasiliev A. A. ${ }^{2}$, Mourenas D. ${ }^{4}$ \\ 1 Institute of Geophysics and Planetary Physics, University of California, \\ Los Angeles, CA, USA, ${ }^{2}$ Space Research Institute, \\ Moscow, Russia, ${ }^{3}$ Department of Mathematical Sciences, \\ Loughborough University, Loughborough LE11 3TU, \\ United Kingdom, ${ }^{4}$ CEA, DAM, DIF, Arpajon, France
}

(Dated: January 18, 2017)

\begin{abstract}
In this paper we provide a theoretical model describing the evolution of the charged particle distribution function in a system with nonlinear wave particle interactions. Considering a system with strong electrostatic waves propagating in an inhomogeneous magnetic field, we demonstrate that individual particle motion can be characterized by the probability of trapping into the resonance with the wave and by the efficiency of scattering at resonance. These characteristics, being derived for a particular plasma system, can be used to construct a kinetic equation (or generalized FokkerPlanck equation) modelling the long-term evolution of the particle distribution. In this equation, effects of charged particle trapping and transport in phase space are simulated with a nonlocal operator. We demonstrate that solutions of the derived kinetic equations agree with results of test particle tracing. The applicability of the proposed approach for the description of space and laboratory plasma systems is also discussed.
\end{abstract}

PACS numbers: 52.35.-g, 52.20.Dq, 96.50.Fm

\section{INTRODUCTION}

The relaxation of unstable plasma distributions in collisionless space plasma systems is realized through plasma wave generation and the following charged particle scattering by these waves. This is a fundamental process, responsible for many phenomena in space and laboratory plasmas. One of the brightest examples is electron acceleration in radiation belts, which results in aurora formation $[1,2]$ and represents a real hazard for artificial satellites in activity [3]. Assuming that the wave spectrum is wide enough and the wave intensity is sufficiently low, one can model the wave-particle resonant interactions using quasi-linear theory [4-7] generalized for systems with inhomogeneous magnetic field and background plasma $[8,9]$. However, various recent spacecraft observations in the near-Earth plasma environment [10-14], laboratory experiments $[15,16]$, and inertial confinement fusion simulations and experiments [17-19] have demonstrated that the applicability of quasi-linear theory is often questionable, because electrons happen to interact with coherent waves sufficiently intense to significantly influence electron motion over long time intervals. There are two main effects of such nonlinear wave-particle interactions: particle trapping by the waves (phase trapping) and particle scattering (phase bunching) with nonzero average change of particle energy [20-22].

The theoretical description of nonlinear wave-particle interaction is based on analysis of individual particle trajectories resonating with waves [23-26] or on a dynamical system approach [27, 28]. These analyses provide all characteristics of particle acceleration and scattering [29-31]. Currently, results derived from this test particle approach are widely used for predictions of charged particle acceleration [32-36] and nonlinear wave gener- ation [37-39]. However, a crucial and still unresolved issue is how to include the characteristics of individual short-term particle interactions with intense waves into a kinetic equation describing the full evolution of the particle distribution function.

Rapid particle transport in phase space via trapping by intense waves cannot be described by differential operators. Thus, integral operators were proposed to include them into a kinetic equation [40-42] to take this process into account. However, in many plasma systems with nonlinear wave-particle interaction, trapping effects are somehow compensated by effects of nonlinear scattering $[35,43,44]$, i.e. the rapid acceleration of a small population of trapped particles corresponds to a slight energy decrease for a large population of scattered particles. This fine balance may allow some intense wave to propagate in an inhomogeneous plasma without significant damping [22]. Therefore, effects of trapping and scattering should be included into the kinetic equation with a proper treatment of the relationship between these two processes. A possible approach for constructing such a model was proposed in [45], where a generalized FokkerPlanck equation has been derived in a simplified but illustrative case. This approach is based on the recognition that trapping is a probabilistic process, i.e., that one can introduce the probability for an individual particle to get trapped during one passage through the resonance [4649]. In such a case, the probability of trapping can be shown to be directly related to the drift in a phase space due to nonlinear scattering [45].

As a significant improvement over our previous preparatory work [45], we consider here a complex system resembling many realistic plasma systems - e.g., interaction of electrons with an electrostatic whistler-mode wave [50] - and combine several earlier results to derive a generalized kinetic Fokker-Planck equation properly de- 
scribing the dynamics of this complex system. Namely, we apply to this system the methods proposed in [35] to derive an analytical expression for the drift velocity $V_{h}$ due to particle nonlinear scattering and the methods from [51] to evaluate the probability of trapping $\Pi$; the proof of the relationship between $V_{h}$ and $\Pi$ provided in [45] is similarly used to construct a generalized Fokker-Planck equation for the particle distribution function. The combination of these three main results allows us to describe for the first time (to the best of our knowledge) a realistic plasma system with nonlinear wave-particle interaction by means of a kinetic equation. To provide a detailed description, we first repeat some elements of previous investigations, e.g., we demonstrate that the derived analytical equations for trapping probability and efficiency of scattering work accurately. Next, these analytical equations are used to derive the generalized Fokker-Planck equation describing the long-term evolution of the full particle distribution function. Solutions of this equation are finally shown to be in good agreement with results of test particle simulations.

\section{HAMILTONIAN EQUATIONS}

We consider non-relativistic charged particle motion (mass $m$, charge $e$ ) in an inhomogeneous magnetic field $B(s)$, where $s$ is a field-aligned coordinate. The system also contains an electrostatic wave propagating along this magnetic field. Thus, transverse particle motion (gyrorotation around magnetic field) is not perturbed by the wave field-aligned electric field, and we can use a guidingcenter approximation for the particle Hamiltonian [52]:

$$
\mathcal{H}=\frac{1}{2 m} p_{\|}^{2}+\mu B(s)+e \Phi(s) \sin \phi
$$

where $p_{\|}$is the particle momentum conjugated to $s, \mu$ is the magnetic moment, wave scalar potential amplitude is $\Phi(s)=\Phi_{0} u(s)$ (function $u<1$ describing the distribution of wave field along a magnetic field line). For a constant wave phase velocity, the phase $\phi$ can be written as $\phi=k_{0} s-\omega t$ ( $k_{0}$ being the wave number and $\omega$ the wave frequency). Using typical energy $\mathcal{H}_{0}$ and spatial scale of background magnetic field variation $R$, we introduce dimensionless variables: $p=p_{\|} / \sqrt{\mathcal{H}_{0} m}, z=s / R$ (thus, time is normalized to $R / \sqrt{\mathcal{H}_{0} / m}$ ) $H=\mathcal{H} / \mathcal{H}_{0}$. We normalize the magnetic field $B(z)$ to its minimum value $B_{0}$, yielding $b=B / B_{0}$. Thus, the dimensionless frequency of particle oscillations along magnetic field lines is: $\Omega_{b}=\sqrt{\mu B_{0} / \mathcal{H}_{0}}$. Wave amplitude is normalized as $\varepsilon=e \Phi_{0} / \mathcal{H}_{0}$. We also introduce the dimensionless wave number $k=k_{0} R$ and dimensionless wave phase velocity $v_{\phi}=\omega \sqrt{m / \mathcal{H}_{0}} / k$.

The new dimensionless Hamiltonian $H$ describing particle oscillations in the $(z, p)$ plane with frequency $\Omega_{b}$ and interaction with an electrostatic wave propagating along $z$ with velocity $v_{\phi}=$ const takes the form

$$
\begin{aligned}
H & =\frac{1}{2} p^{2}+\frac{1}{2} \Omega_{b}^{2} b(z)+\varepsilon u(z) \sin \phi \\
\phi & =k\left(z-v_{\phi} t\right)
\end{aligned}
$$

We further consider a background magnetic field with a spatial scale $R$ much larger than the wavelength, i.e. $k=k_{0} R \gg 1$. We expand the dimensionless magnetic field around its minimum value at $z=0$ and keep the three first terms of this expansion: $b(z)=1+z^{2}+b_{0} z^{4} / 2$ (see discussion for explanations why $b_{0} \neq 0$ is important). Wave amplitude is taken as much smaller than typical particle energy $\varepsilon \ll 1$ (i.e., the wave field perturbs only slightly particle motion along the almost entire particle trajectory). We also assume the following relationship between the two small parameters $\varepsilon$ and $1 / k: \varepsilon \sim 1 / k$. We consider here realistic electrostatic waves generated at the magnetic field minimum $z=0$ and propagating along magnetic field lines $[82,88]$. Therefore, the wave amplitude $\sim u$ grows with $|z|: u=\left(\tanh (\tilde{z})-\tanh \left(\tilde{z}_{0}\right)\right) / 2$ for $z \geq 0$, with $\tilde{z}=\left(z-z_{0}\right) / l, \tilde{z}_{0}=-z_{0} / l, z_{0}=1$, and $l=3$ (for the sake of simplicity, we further take $u=0$ for $z<0)$.

Two examples of solutions of Hamiltonian equations for Hamiltonian (1) are shown in Fig. 1. Far from the resonance $\dot{\phi}=0$, particles move along regular closed trajectories in the $(z, p)$ plane. These trajectories correspond to particle oscillations in the potential $\sim \Omega_{b}^{2} b(z) / 2$ with frequency $\sim \Omega_{b}$. High-frequency waves $\left(k v_{\phi}=\omega \gg \Omega_{b}\right)$ cannot disturb this motion. However, in the vicinity of the resonance $\dot{\phi}=0$, the wave phase $\phi$ changes at a speed comparable with particle velocity of motion. Therefore, particle motion can be significantly disturbed by the wave field. There are two main effects corresponding to such a disturbance: scattering and trapping. The left panels of Fig. 1 show examples of scattering when particle energy is slightly changed during the resonance crossing. This change consists of a nonzero mean value and a stochastic part. In case of many passages through the resonance, scattering can result in diffusive energy variations (or/and energy drift). The right panels of Fig. 1 show an example of particle trapping by the wave field. Trapped particles have their characteristic motion essentially modified and start moving with the wave (i.e. $p \approx v_{\phi}$ ). Trapped motion stops when a particle escapes from the resonance. During such a trapped motion, particle energy changes regularly as $z \sim v_{\phi} t$ and $p \approx v_{\phi}$. To describe both scattering and trapping processes for a larger particle ensemble, we start with the consideration of a single particle motion near the resonance.

To study resonant effects in Hamiltonian (1), we follow the standard procedure [54]: (1) introduce the fast phase $\phi$ as a new variable; (2) expand the Hamiltonian around the close vicinity of the resonance $\dot{\phi}=0 ;(3)$ separate the expanded Hamiltonian into two parts describing fast oscillations of $\phi$ near the resonance and slow $(z, p)$ change. The analysis of the fast Hamiltonian provides us with local system characteristics (amplitudes of scat- 

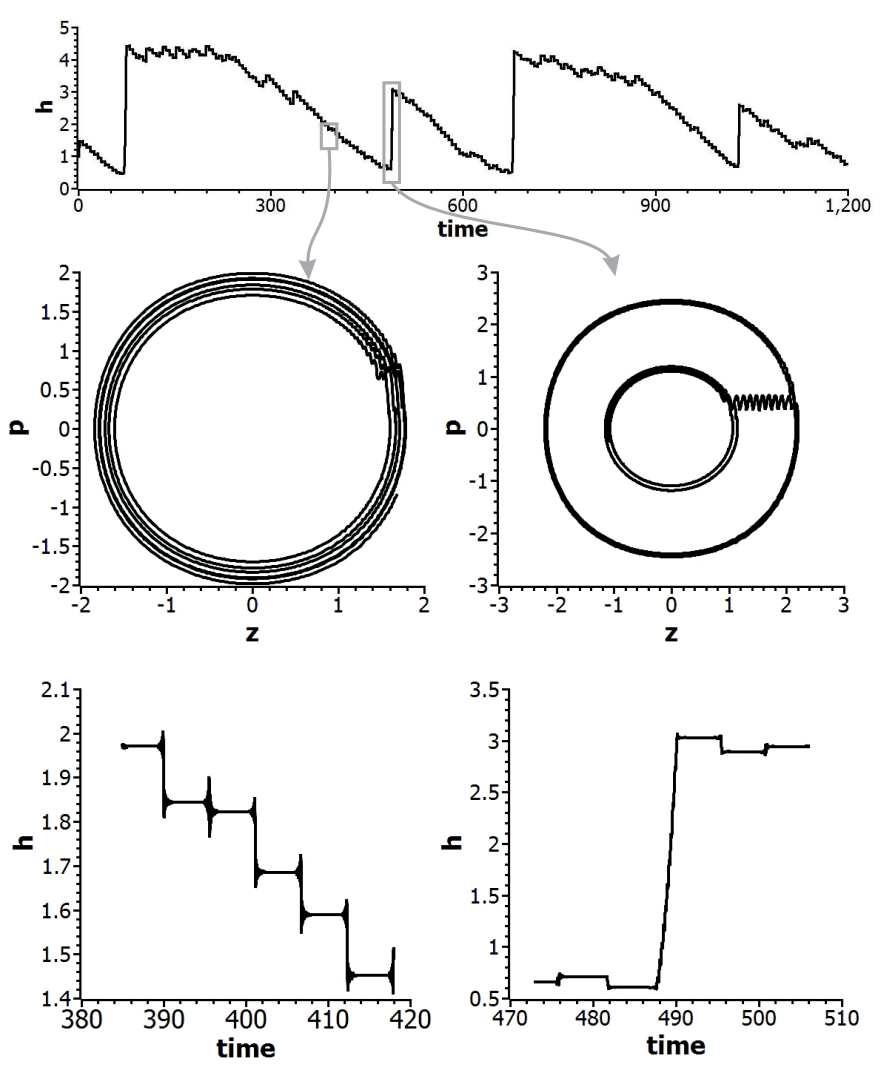

FIG. 1: Particle trajectory: top panel shows the long-term evolution of particle energy $h$, middle and bottom panels show fragments of trajectory for scattering (left) and trapping (right). System parameters are: $\Omega_{b}=1, v_{\phi}=0.5, k=100$,

$\tilde{b}_{0}=2 h_{0} b_{0} / \Omega_{b}^{2}=0.1, \varepsilon=0.05$, and initial particle energy equals $h_{0}=\sqrt{2}$.

tering, probability of trapping, etc.), whereas the analyse of the slow Hamiltonian describes energy variation of trapped particles.

To introduce the wave phase $\phi$ as a new variable, we use the generating function $W=I(k z-\omega t)+\tilde{p} z$ where $\omega=k v_{\phi} \sim k \gg 1$ and $(\tilde{p}, \tilde{z})$ are new variables:

$$
p=\frac{\partial W}{\partial z}=\tilde{p}+k I, \quad z=\frac{\partial W}{\partial \tilde{p}}=\tilde{z}
$$

The corresponding new Hamiltonian $\tilde{H}=H+\partial W / \partial t$ takes the form:

$$
\tilde{H}=-\omega I+\frac{1}{2}(\tilde{p}+k I)^{2}+\frac{1}{2} \Omega_{b}^{2} b(\tilde{z})+\varepsilon u(\tilde{z}) \sin \phi
$$

We omit tildes in $\tilde{p}$ and $\tilde{z}$, and write the equations of motion:

$$
\begin{aligned}
& \dot{i}=-k \frac{\partial \tilde{H}}{\partial \phi}=-k \varepsilon u(z) \cos \phi \\
& \dot{\phi}=\frac{\partial \tilde{H}}{\partial I}=-\omega+k(p+i)
\end{aligned}
$$

$$
\begin{aligned}
& \dot{p}=-\frac{\partial \tilde{H}}{\partial z}=-\frac{1}{2} \Omega_{b}^{2} b^{\prime}(z)-\varepsilon u^{\prime}(z) \sin \phi \\
& \dot{z}=\frac{\partial \tilde{H}}{\partial p}=p+i
\end{aligned}
$$

where $i=k I$. Equations (3) show that $\phi$ changes much faster than $(z, p, i)$ (because $\dot{\phi} \sim k)$. The resonant condition $-\omega+k(p+i)=0$ gives the resonant $i_{R}=v_{\phi}-p$. We expand Hamiltonian $\tilde{H}$ around the resonant surface $I_{R}=i_{R}(z, p) / k$ :

$$
\tilde{H} \approx \Lambda(z, p)+\frac{1}{2} g(z, p)\left(I-I_{R}\right)^{2}+\varepsilon u(z) \sin \phi
$$

where

$$
\begin{aligned}
\Lambda(z, p) & =-v_{\phi} i_{R}+\frac{1}{2}\left(p+i_{R}\right)^{2}+\frac{1}{2} \Omega_{b}^{2} b(z) \\
& =v_{\phi} p+\frac{1}{2} \Omega_{b}^{2} b(z)-\frac{1}{2} v_{\phi}^{2}
\end{aligned}
$$

and $g=\partial^{2} \tilde{H} / \partial I^{2}$ is evaluated at $I=i_{R} / k\left(g=k^{2}\right)$. We introduce a new momentum $K=I-I_{R}$ with the generating function $W_{1}=\bar{p} z+\left(K+I_{R}\right) \phi$ (where $(\bar{z}, \bar{p})$ are new variables):

$$
\begin{aligned}
\bar{H} & \approx \Lambda(\bar{z}, \bar{p})+\frac{1}{2} k^{2} K^{2}+\varepsilon u(\bar{z}) \sin \phi+\left\{I_{R}, \Lambda\right\} \phi \\
& =\Lambda(\bar{z}, \bar{p})+\frac{1}{2} k^{2} K^{2}+\frac{1}{2} \Omega_{b}^{2} \frac{b^{\prime}(\bar{z})}{k} \phi+\varepsilon u(\bar{z}) \sin \phi
\end{aligned}
$$

We introduce the Hamiltonian $F=\bar{H}-\Lambda$ and omit bars in $(\bar{z}, \bar{p})$ :

$$
\begin{aligned}
& \bar{H}=\Lambda(z, p)+F \\
& F=\frac{1}{2} k^{2} K^{2}+\frac{1}{2} \Omega_{b}^{2} \frac{b^{\prime}(z)}{k} \phi+\varepsilon u(z) \sin \phi
\end{aligned}
$$

where $k \varepsilon \sim 1$. The Hamiltonian $F$ describes fast $(\dot{\phi} \sim k)$ oscillations of wave phase $\phi$, whereas the Hamiltonian $\Lambda(z, p)$ describes slow variation of $(z, p)$. For $\Omega_{b}^{2} b^{\prime}(z)<$ $2 k \varepsilon u(z)$ there is a region in the phase plane $(\phi, K)$ filled with closed trajectories (see Fig. 2). The corresponding area of this region is

$$
\begin{aligned}
S & =2 \int_{\phi_{1}}^{\phi_{\max }} K d \phi=\sqrt{\frac{8}{k^{3}}} \int_{\phi_{1}}^{\phi_{\max }} \sqrt{k F_{s}-\frac{1}{2} \Omega_{b}^{2} b^{\prime} \phi-k \varepsilon u \sin \phi} d \phi \\
& =\sqrt{\frac{4 \Omega_{b}^{2} b^{\prime}}{k^{3}}} \int_{\phi_{1}}^{\phi_{\max }} \sqrt{\phi_{\max }-\phi+a\left(\sin \phi_{\max }-\sin \phi\right)} d \phi \\
& =\frac{R(z)}{k^{3 / 2}}
\end{aligned}
$$

where $F_{s}$ is the value of $F$ at the saddle point (see Fig. 2), $\phi_{1}$ and $\phi_{\max }$ are shown in Fig. 2, and $a=$ $2 k \varepsilon u(z) / \Omega_{b}^{2} b^{\prime}(z)$. Particles moving along closed trajectories oscillate around the resonance $\dot{\phi}=0$. Such trapped particles propagate with the wave. In contrast, transient 


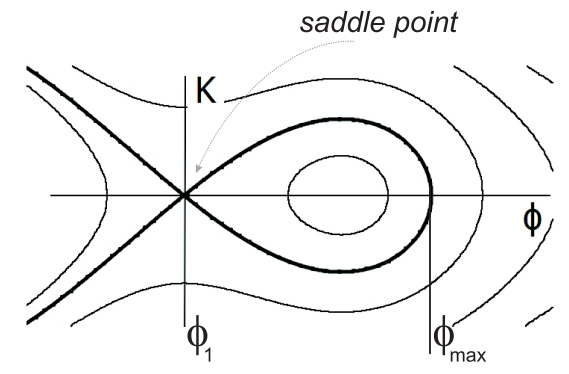

FIG. 2: Phase portrait of system (7).

particles moving along open trajectories cross the resonance in a short time interval and can merely be scattered by waves. To characterize resonant interaction of a wave with a particle ensemble, we should describe the evolution of the energy of trapped particles, the energy variation of transient particles, as well as the transitions between transient and trapped populations. These transitions correspond to a transient particle getting trapped into resonance and a trapped particle escaping from the resonance. During trapped particle motion, its phase $\phi$ and corresponding conjugated moment $K$ oscillate much faster than $(z, p)$ change. Thus, the area $I_{\phi}$ surrounded by a closed trajectory in $(\phi, K)$ plane can be considered as an adiabatic invariant [53]. This invariant is equal to the area $S$ at the moment of capture into trapping. Conservation of $I_{\phi}$ guarantees that trapped particle escapes from the resonance when a decreasing $S$ returns to a value equal to $I_{\phi}$, i.e. equal to the value at the moment of trapping. Therefore, particle escape from the resonance can be described using the time profile $S(z, p)=S(t)$ along the resonant trajectory, i.e. when $(z, p)$ evolution is described by the Hamiltonian $\Lambda$. Trapping into resonance also depends on the $S(t)$ profile: to be trapped, a particle should approach the resonance when $S$ increases. We describe the process of trapping-escape and corresponding energy variations in section II A, whereas section II B is devoted to energy variation for transient particles scattered in the resonance.

\section{A. Trapping}

The number of transient particles crossing the separatrix and becoming trapped during a short time interval $\Delta t$ equals to the product of $\Delta t$ and $\dot{S}$. The ratio of $\Delta t \dot{S}$ to the total phase space volume passing through the resonance $\Delta t \dot{\mathcal{W}}$ with $\mathcal{W}=\left|\int_{0}^{2 \pi} K d \phi\right|$ can be defined as a probability of trapping $\Pi[47]$. This probability is equal to the relative number of particles which will get trapped during one passage through the resonance. The area $S$ depends only on $z$, thus we have $\dot{S}=\dot{z}(\partial S / \partial z)$ where $\dot{z}=v_{\phi}$ at resonance. The phase space flux $\dot{\mathcal{W}}=\left|\int_{0}^{2 \pi} \dot{K} d \phi\right|$ can be calculated as:

$$
\dot{\mathcal{W}}=\left|\int_{0}^{2 \pi} \dot{K} d \phi\right|=\pi \Omega_{b}^{2} \frac{\left|b^{\prime}(z)\right|}{k}
$$

where we take into account the Hamiltonian equation for $K$ from Hamiltonian (7). Therefore, we can write the following expression for $\Pi=\dot{S} / \mathcal{W}$ :

$$
\Pi=\frac{1}{\pi} \frac{k v_{\phi}}{\Omega_{b}^{2}\left|b^{\prime}(z)\right|} \frac{\partial S}{\partial z}=\frac{1}{\pi} \frac{v_{\phi}}{\Omega_{b}^{2}\left|b^{\prime}(z)\right| \sqrt{k}} \frac{\partial R(z)}{\partial z}
$$

Equation (10) should be evaluated at the resonant value of $z=z_{R}$ :

$$
z_{R}=\sqrt{\frac{-1+\sqrt{1+2 b_{0} \Omega_{b}^{-2}\left(2 h-v_{\phi}^{2}\right)}}{b_{0}}}
$$

where $h$ is the particle energy (1) before resonant interaction with the wave. Substituting Eq. (11) into Eq. (10), we obtain $\Pi=\Pi(h)$. Thus, for each given set of parameters $v_{\phi}, k, \Omega_{b}$, and $\varepsilon$, we can evaluate the analytical $\Pi$ and compare it with numerical calculations. We calculate the numerical probability of trapping as the ratio of particles trapped during one passage through the resonance to the total number of resonant particles (we integrate numerically $10^{6}$ trajectories). Figure 3 shows that Eq. (10) describes the probability of trapping quite well.

As trapped particles move with the wave $\left(p \approx v_{\phi}\right)$, they gain energy. Then, after some time, trapped particles escape from the resonance (see Fig. 1). To calculate the amount of energy gained by trapped particles before their escape from resonance, one should consider the evolution of $S$ along the trapped particle trajectory. The coordinate corresponding to particle escape from resonance $z_{e s c}$ can be found from the equation

$$
S\left(z_{\text {esc }}\right)=S\left(z_{\text {trap }}\right), \quad \partial S /\left.\partial z\right|_{z=z_{\text {esc }}}<0
$$

where $z_{\text {trap }}$ is the coordinate at the time of trapping. Knowing $z_{e s c}$, one can calculate the energy gained by particles:

$$
\Delta h=\frac{1}{2} v_{\phi}^{2}+\frac{1}{2} \Omega_{b}^{2} b\left(z_{e s c}\right)-h=\frac{1}{2} \Omega_{b}^{2}\left(b\left(z_{e s c}\right)-b\left(z_{\text {trap }}\right)\right)
$$

where $h$ is the particle energy before resonant interaction with the wave. We have calculated the energy gain given by (13) and compared it with numerical results. Figure 4 shows that particle acceleration is correctly described by (13). Therefore, we can calculate analytically both the number of trapped particles and the energy that these particles can gain.

\section{B. Scattering}

In this subsection, we consider the scattering of resonant particles (see one example of a scattered particle 

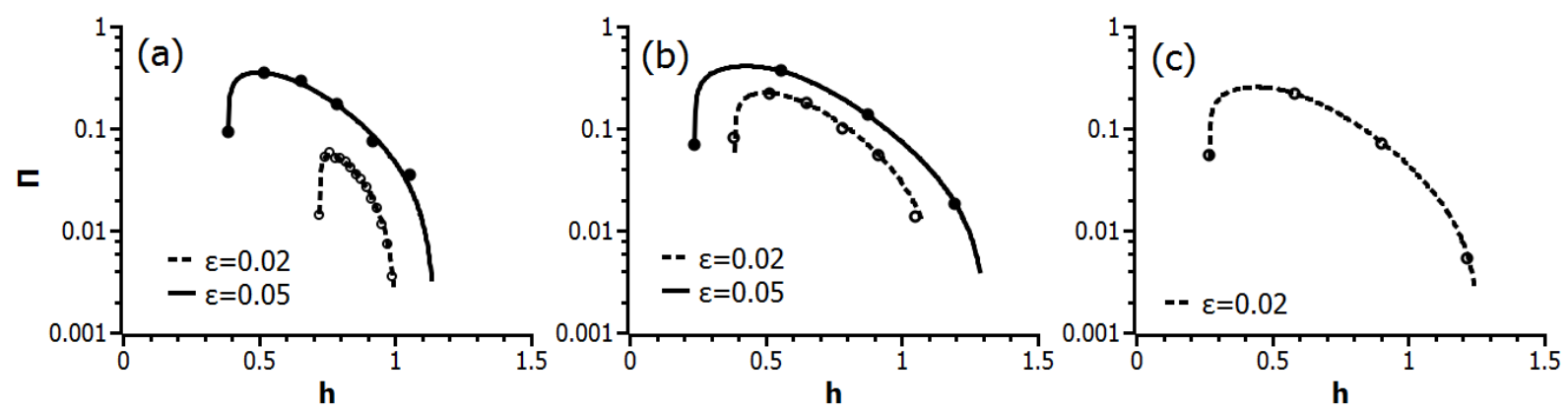

FIG. 3: Probability of trapping: numerical (dots) and analytical (curves) results. System parameters are: $\Omega_{b}=1$, $v_{\phi}=0.5, \tilde{b}_{0}=0.1$. Different panels show result for different $k$ : (a) $k=100$, (b) $k=250$, (c) $k=500$.
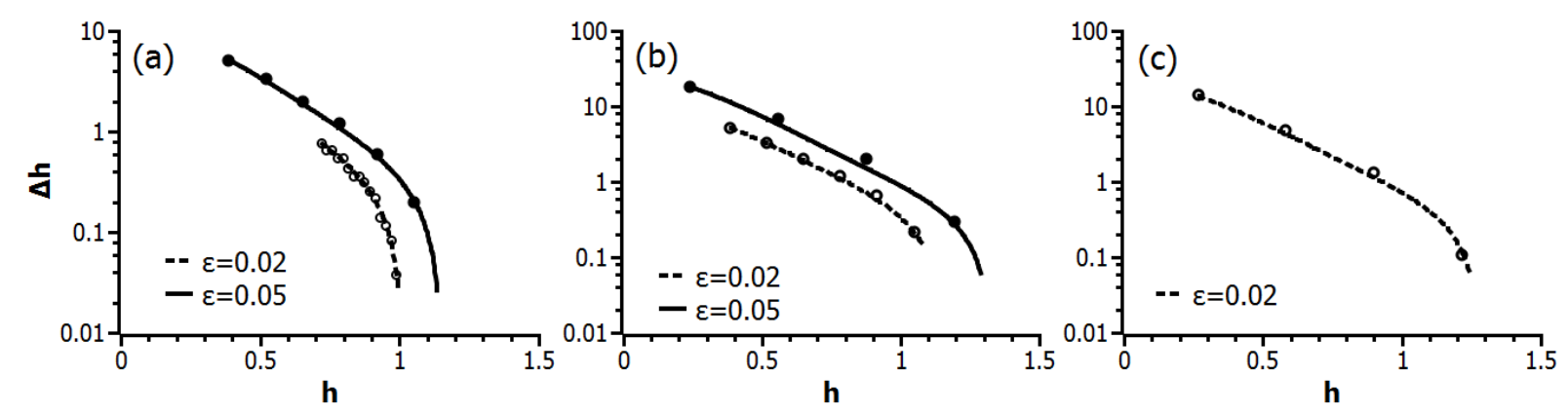

FIG. 4: Energies gained by trapped particles: numerical (dots) and analytical (curves) results. System parameters are: $\Omega_{b}=1, v_{\phi}=0.5, \tilde{b}_{0}=0.1$. Different panels show result for different $k:(\mathrm{a}) k=100,(\mathrm{~b}) k=250,(\mathrm{c}) k=500$.

trajectory in Fig. 1, left panel). Passage through the resonance results in variation of momentum $I$ (see Eqs. $(3))$ :

$$
\begin{aligned}
\Delta I & =2 \int_{-\infty}^{t_{*}} \dot{I} d t=-2 \varepsilon u(z) \int_{-\infty}^{\phi_{*}} \frac{\cos \phi}{\dot{\phi}} d \phi \\
& =-\frac{\sqrt{2} \varepsilon u(z) k^{2}}{\sqrt{k}} \int_{-\infty}^{\phi_{*}} \frac{\cos \phi d \phi}{\sqrt{F k-\Omega_{b}^{2} b^{\prime} \phi / 2-k \varepsilon u(z) \sin \phi}} \\
& =-\sqrt{2 \varepsilon u(z)} k \int_{-\infty}^{\phi_{*}} \frac{a^{1 / 2} \cos \phi}{\sqrt{2 \pi \theta-\phi-a \sin \phi}} d \phi \\
& =\sqrt{2 \varepsilon u(z)} k Q(\theta, a)
\end{aligned}
$$

where $a=2 k \varepsilon u\left(z_{R}\right) / \Omega_{b}^{2} b^{\prime}\left(z_{R}\right), 2 \theta=k F / \Omega_{b}^{2} b^{\prime}\left(z_{R}\right)$, and $\phi_{*}=\phi_{*}(\theta, a)$ is the solution of equation $2 \pi \theta-\phi-a \sin \phi=$ $0\left(\phi_{*}\right.$ is the value of $\phi$ at time $t_{*}$ when the particle crosses the resonance $\dot{\phi}=0)$. The profile of function $Q(\theta, a)$ is displayed in Fig. 5. Function $Q$ is periodic over $\theta$ with period 1 . The resonant phase $\phi_{*}$ depends on initial phase value far from the resonance. The phase $\phi$ changes fast far from $\dot{\phi}=0$ and even a small change of the initial phase can significantly change $\phi_{*}$. Therefore, it is reasonable to consider the average $\Delta I$. Averaging should be performed over $\theta$ :

$$
\begin{aligned}
\langle\Delta I\rangle & =\sqrt{2 \varepsilon u} k\langle Q\rangle_{\theta} \\
\operatorname{Var}(\Delta I) & =2 \varepsilon u k^{2}\left(\left\langle Q^{2}\right\rangle_{\theta}-\langle Q\rangle_{\theta}^{2}\right)=2 \varepsilon u k^{2} \operatorname{Var}(Q)
\end{aligned}
$$

where $z$ is evaluated at resonance. Figure 6 shows both functions $\langle Q\rangle_{\theta}$ and $\operatorname{Var}(Q)$. The average value $\langle Q\rangle_{\theta}$ is equal to zero for $a<1$, i.e. for small wave amplitude we have only a diffusive scattering without any drift in $I$ space.

The jump $\Delta I$ is related to the jump of energy $(-\omega I+$ $h=$ const, see Eqs. $(1,2))$ :

$$
\Delta I=\Delta h / \omega=\Delta h /\left(k v_{\phi}\right)
$$

Thus, we can write

$$
\langle\Delta h\rangle=\sqrt{2 \varepsilon v_{\phi}^{2} u}\langle Q\rangle_{\theta}, \operatorname{Var}(\Delta h)=2 \varepsilon v_{\phi}^{2} u \operatorname{Var}(Q)
$$

where $u$ and $Q$ are evaluated at $z=z_{R}$. We calculate analytical changes of energy due to scattering (17) and numerical changes. Figure 7 shows that both drift $\langle\Delta h\rangle$ and variance $\operatorname{Var}(\Delta h)$ given by Eq. (17) describe numerical results rather well. 


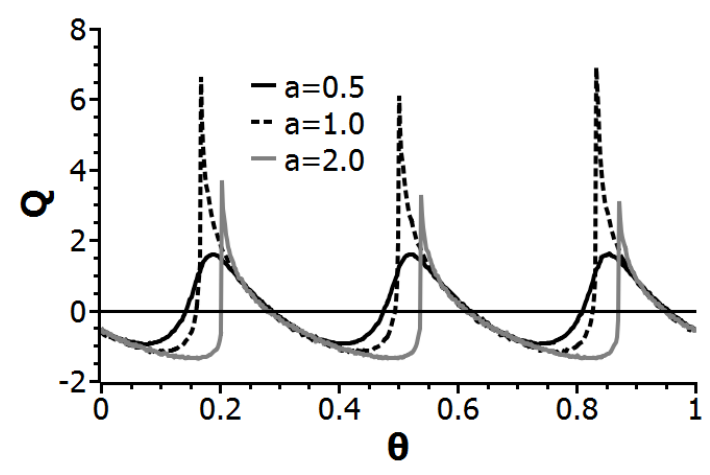

FIG. 5: Three profiles of $Q(\theta, a)$ function.

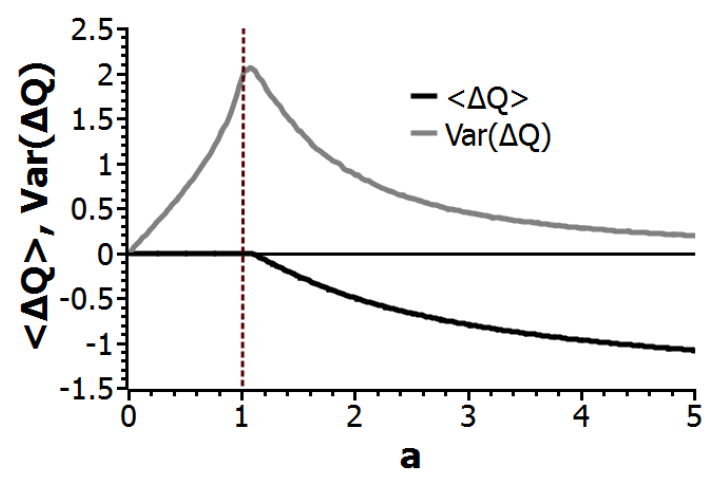

FIG. 6: Profiles of $\langle Q\rangle_{\theta}$ and $\operatorname{Var}(Q)$.

\section{EVOLUTION OF THE DISTRIBUTION FUNCTION}

Equations derived in sections II A, II B provide all the necessary information for modeling the evolution of a large particle ensemble. Now, we introduce the distribution function of particle moment $f(I)$. For a system with trapping and scattering, the kinetic equation for this function was derived in [45]:

$$
\frac{\partial f}{\partial t}=\frac{\partial}{\partial I}\left(D_{I I} \frac{\partial f}{\partial I}\right)-V_{I} \frac{\partial f}{\partial I}-\frac{d V_{I}}{d I}\left(f-f_{*}\right) \Theta(I)
$$

where $D_{I I}=\left\langle\Delta I^{2}\right\rangle / 2 \tau_{0}$ (where $\left\langle\Delta I^{2}\right\rangle=\operatorname{Var}(\Delta I)+$ $\left.\langle\Delta I\rangle^{2}\right), V_{I}=\langle\Delta I\rangle / \tau_{0}, \Theta(I)$ is equal to one for $I$ with $\Pi=0$ and to zero for $I$ with $\Pi>0$. The function $f_{*}$ is equal to $f\left(I_{*}\right)$ where $I_{*}$ is the value that a particle should have when it gets trapped in order to escape from the resonance with $I$. Equation (18) was derived for constant period of particle oscillations between two passages of resonances $\tau_{0}=$ const and should be generalized for our system with $\tau_{0}(I)$. Equation (18) describes the evolution of the distribution $f(I)$ on a time scale much larger than $\tau_{0}$, where $\tau_{0}(I)$ is the time between two successive resonant interactions. The first two terms in Eq. (18) describe particle scattering by an intense wave. If the wave amplitude is not sufficiently large to provide $a>1$, the drift velocity $V_{I}$ drops to zero (see Fig. 6) and only the diffusion term $\sim D_{I I}$ provides some variation of the particle distribution $f(I)$. The last term in Eq. (18) describes particle transport in $I$-space due to trapping. To write this term in the present form, we employ the useful equality derived in [45]: $\Pi=-d V_{I} / d I$. This equality describes the relation between probability of trapping and drift velocity and it is based on a very important property of the integral (14): $\langle\Delta I\rangle_{\theta}=-\operatorname{sign}(a) S / 2 \pi[54,55]$. The term $\sim\left(f-f_{*}\right)$ is nonlocal, because it describes the change of particle distribution at $I$ as a function of the particle distribution at $I_{*}$. Thus, Eq. (18) describes the nonlocal probabilistic process of particle trapping and transport in $I$-space.

We start with a generalization of Eq. (18) for systems with $\tau_{0}=\tau_{0}(I)$ and then rewrite it for a distribution function of energy $h$. The definition of the diffusion term allows us to keep the first term in Eq. (18) with $D_{I I}=$ $\left\langle\Delta I^{2}\right\rangle / 2 \tau_{0}(I)$. However, nonlinear terms $\sim V_{I}, \sim d V_{I} / d I$ should be rewritten. Omitting terms $\sim d \ln \tau_{0} / d I(\tau(I)$ changes insignificantly in our system, see Fig. 8, left panel), we can rewrite Eq. (18) as

$$
\frac{\partial f}{\partial t}=\frac{\partial}{\partial I}\left(D_{I I} \frac{\partial f}{\partial I}\right)-V_{I} \frac{\partial f}{\partial I}-\frac{d V_{I}}{d I}\left(f-f_{*} \frac{\tau_{0}}{\tau_{0}^{*}}\right) \Theta(I)
$$

where $\tau_{0}=\tau_{0}(I), V_{I}=\langle\Delta I\rangle / \tau_{0}(I), \tau_{0}^{*}=\tau_{0}\left(I_{*}\right)$.

Equation (19) can finally be rewritten as

$$
\frac{\partial f}{\partial t}=\frac{\partial}{\partial h}\left(D_{h h} \frac{\partial f}{\partial h}\right)-V_{h} \frac{\partial f}{\partial h}-\frac{d V_{h}}{d h}\left(f-f_{*} \frac{\tau_{0}}{\tau_{0}^{*}}\right) \Theta(h)
$$

where we use the relation (16). For a given particle of energy $h$, two successive resonant interactions are separated by a time interval $\tau_{0}(h)=\left(2 \pi / \Omega_{b}\right) T(h)$ with

$$
T(h)=\frac{1}{\pi} \int_{z_{-}}^{z_{+}} \frac{\Omega_{b} d z}{\sqrt{2 h-\Omega_{b}^{2} b(z)}}=\frac{1}{\pi} \int_{y_{-}}^{y_{+}} \frac{d y}{\sqrt{1-y^{2}-\tilde{b}_{0} y^{4} / 2}}
$$

where $\tilde{b}_{0}=2 h b_{0} / \Omega_{b}^{2}$ and $y_{ \pm}$are solutions of equation $1-y^{2}-\tilde{b}_{0} y^{4} / 2=0$. Figure 8 (left panel) shows the variation of $T\left(\tilde{b}_{0}\right)$ with $h$.

Thus, $D_{h h}$ and $V_{h}$ are equal to the ratio of $D_{I I}, V_{I}$ and $\tau_{0}(h)$. We use the expression (11) for the resonant coordinate $z_{R}$ and plot $D_{h h}, V_{h}, d V_{h} / d h$ in Fig. 8 (center panel). To define $h\left(h_{*}\right)$, we solve the equation $h_{*}+\Delta h\left(h_{*}\right)=h$ with $\Delta h$ given by Eq. (13). We also plot $h\left(h_{*}\right)$ in Fig. 8 (right panel).

Using the functions from Fig. 8, we next solve Eq. (20) numerically for three systems with different initial distributions $f_{0}(h)$ and parameters. To check these model solutions, we numerically integrate $10^{6}$ test-particle trajectories and plot the corresponding distributions. The comparison of solutions of Eq. (20) with results of fully numerical tracing demonstrates that Eq. (20) describes well the evolution of the particle distribution (see Fig. 9). It is worth noting that the first numerical verification of 

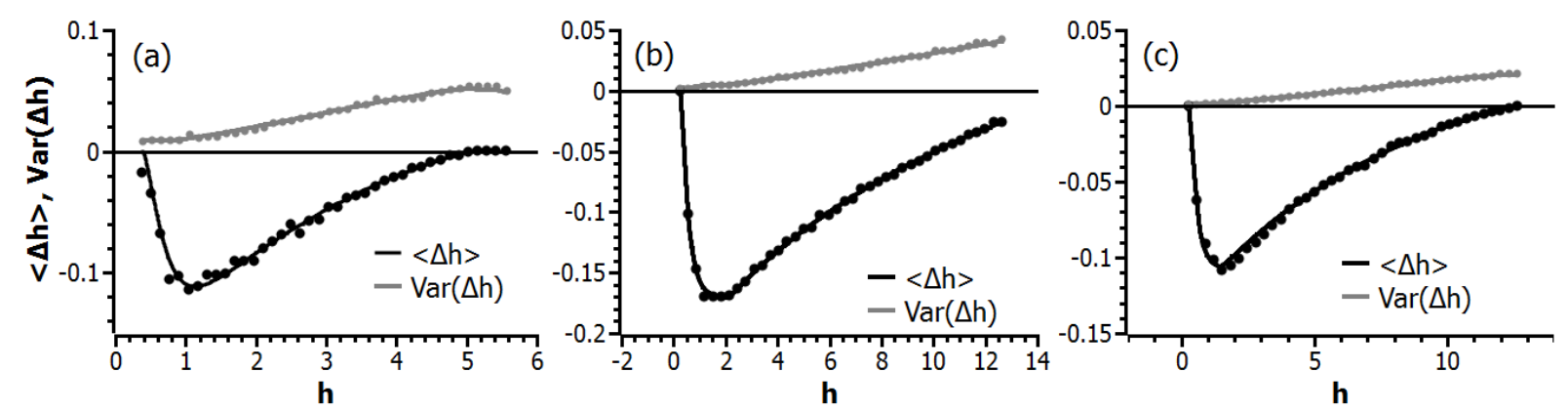

FIG. 7: Energies gained by scattered particles: numerical (dots) and analytical (curves) results. System parameters are: $\Omega_{b}=1, v_{\phi}=0.5, \tilde{b}_{0}=0.1$. Different panels show result for different $k, \varepsilon$ : (a) $k=100$ and $\varepsilon=0.05$, (b) $k=250$ and $\varepsilon=0.05$, (c) $k=500$ and $\varepsilon=0.02$.
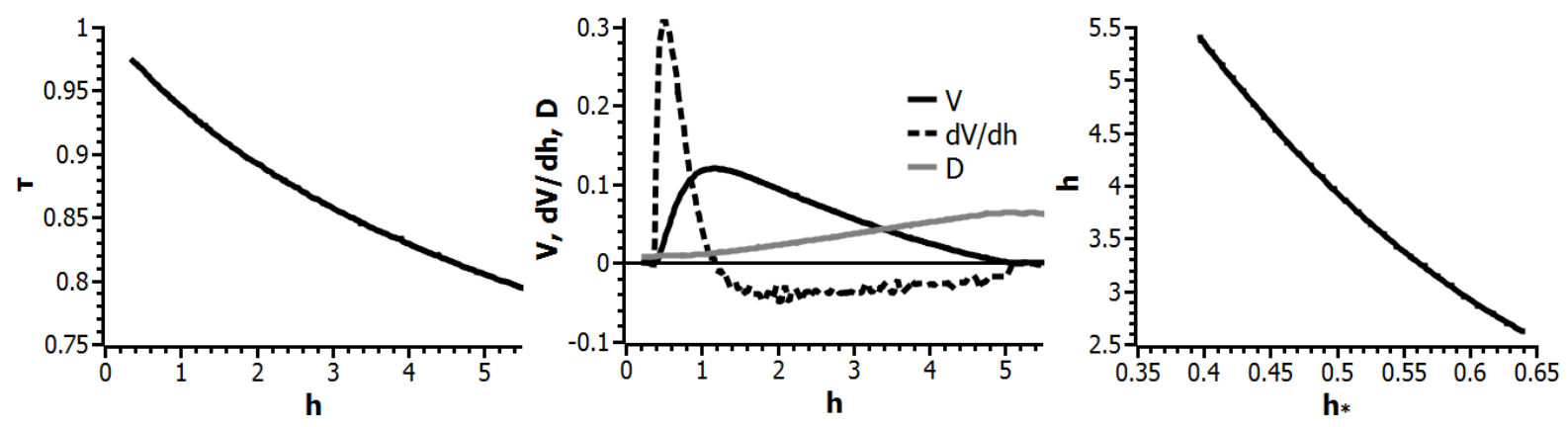

FIG. 8: The period $T$ given by Eq. (21) is shown in the left panel; coefficients of Eq. (20) are displayed in the center panel; the function $h\left(h_{*}\right)$ is shown in the right panel. System parameters are: $\Omega_{b}=1, v_{\phi}=0.5, k=100, \tilde{b}_{0}=0.1$,

$$
\varepsilon=0.05 \text {. }
$$

Eq. (18) was done in [45] for a very simplified (toy) system: model time functions for coefficients in Hamiltonian (7), symmetric space $h^{*}=h_{0}-h$ with $V_{h}(h)=V_{h}\left(h_{0}-h\right)$ and uniform motion $\tau_{0}(h)=$ const. Figure 9 demonstrates the first results for a realistic plasma system.

Figure 9 (left column) shows the evolution of the distribution $f(h)$ in the system with $k=100, \varepsilon=0.05$. The initial distribution $f_{0}(h)$ is localized at small energies $h \in[0.5,1]$ where the probability of trapping is positive (see Fig. 3). Therefore, after only a short time interval, some particle population is already transported to the high energy region $(h>1.5)$, whereas the peak of the distribution drifts to smaller energies due to the negative $V<0$ (see Fig. 7). Particle transport via trapping is very fast and efficient, because the initial distribution has a maximum around the peak value of probability. As a result, after only 10 bounce periods $\left(\sim 2 \pi / \Omega_{b}\right)$ the distribution function already fills all the available energy range and reaches an almost stationary solution when in Eq. (20) both terms $\partial f / \partial h$ and $\sim\left(f-f_{*}\right)$ tend to zero. Such an evolution of the particle distribution corresponds to growth of the average particle energy $\langle h\rangle=\int h f(h) d h / \int f(h) d h$ (see Fig. 10, run \#1). For 10 periods $\langle h\rangle$ increases about three times and then saturates.
A similar evolution of the particle energy distribution can be found in Fig. 9 (middle column), where solutions of Eq. (20) are displayed for system parameters $k=250$, $\varepsilon=0.05$. The larger effective wave amplitude $\sim k \varepsilon$ corresponds to a wider energy range filled by trapped particles ( $h$ reaches $\sim 15$, see Fig. 3, right panel). Saturation of energy growth occurs at the same time (about 10 bounce periods) as for the previous system (compare runs \#1 and \# 2 in Fig. 10).

In contrast to results shown in Figs. 9 (left and middle column), the initial distribution for the run shown in Fig. 9 (right column) is localized at high energy $h \sim 3.5$. For system parameters $k=100, \varepsilon=0.05$, trapping is impossible in the high energy range (see Fig. 3). Thus, particles start drifting to small energies and the average energy $\langle h\rangle$ decreases (see Fig. 9 (right column) and Fig. 10, run \#3). Only when particles reach sufficiently small energies $(h<1)$, does trapping start to transport particles back to the high energy region and the average energy $\langle h\rangle$ increases again (see time larger than 15 bounce periods). Energy saturation occurs only around 25 bounce periods, when the distribution function reaches an almost flat shape. 

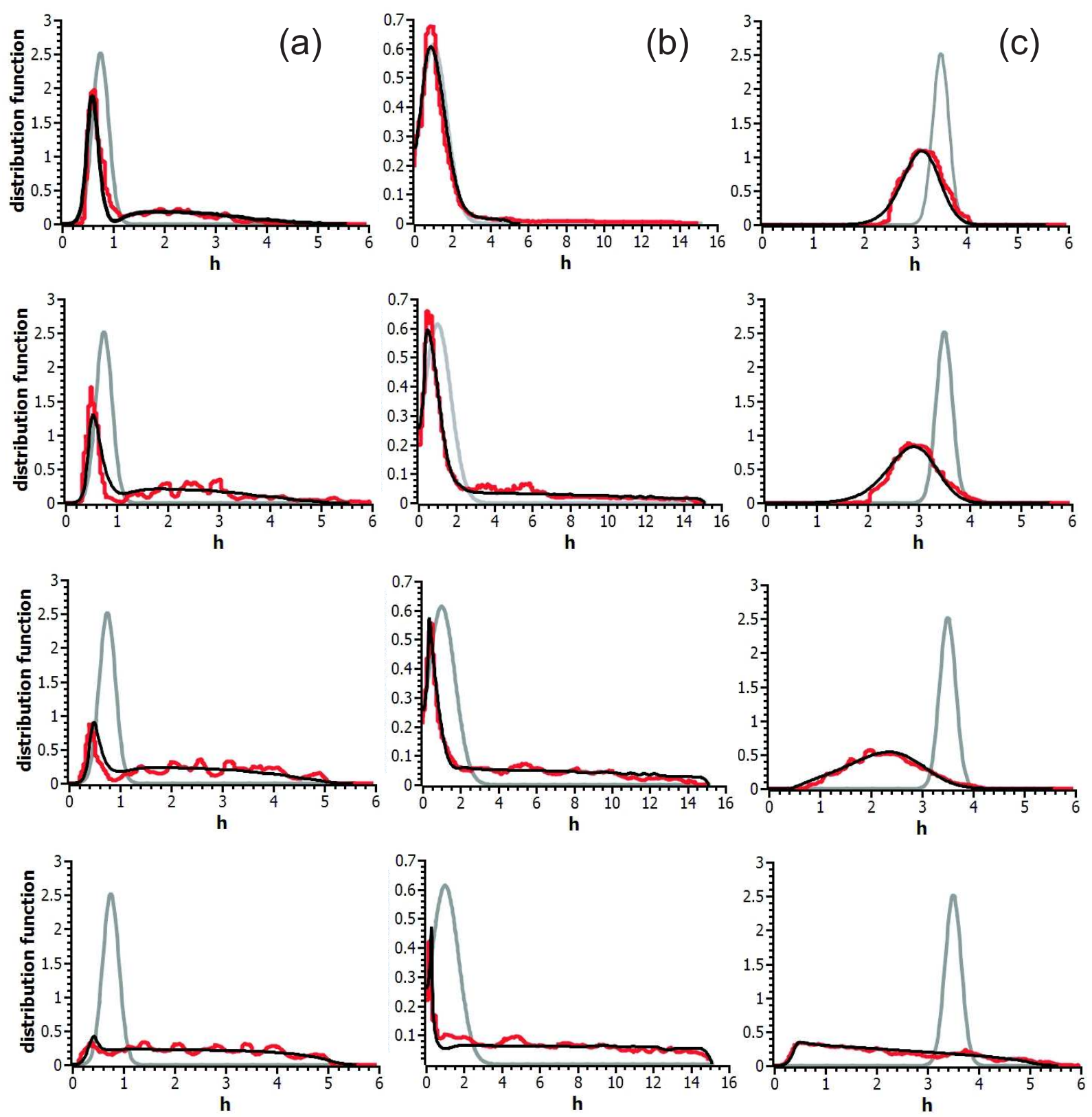

FIG. 9: Particle distributions obtained as a solution of Eq. (20) are shown in black, whereas results of test particle simulations are shown in red. The initial distribution is shown in grey. Time evolution from top to bottom. System parameters are: $\Omega_{b}=1, v_{\phi}=0.5, \tilde{b}_{0}=0.1, \varepsilon=0.05$. Different panels show result for different $k$ and initial distributions: (a) run\# 1 with $k=100$, (b) run\# 2 with $k=250$, (c) run\# 3 with $k=100$. Four time moments are displayed: $t \Omega_{b}=\{1,3,5,10\}$ for runs $\# 1 \& \# 2$ and $t \Omega_{b}=\{3,5,10,25\}$ for run \#3.

\section{CONCLUSIONS}

Equation (20) describes the long-term evolution of the particle distribution in a system with nonlinear waveparticle interaction. This equation can be applied to systems where Landau resonance with electrostatic waves plays an important role. There are several plasma systems in the near-Earth space where such conditions are realized.
First of all, electron acceleration by parallel electric fields of strong kinetic Alfven waves is believed to be responsible for the formation of hot electron field-aligned distributions in the aurora and the equatorial magnetosphere $[56,57]$. Corresponding wave-particle resonant interaction includes both trapping and scattering [58] and, thus, can be modeled by Eq. (20). A second good opportunity for applying the proposed model concerns electron resonant interactions with whistler-mode waves propa- 


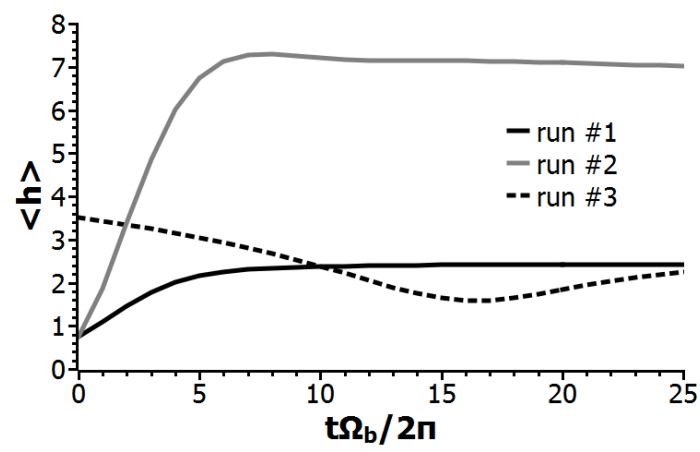

FIG. 10: Average energy $\langle h\rangle$ for three runs from Fig. 9.

gating in a quasi-electrostatic mode in the inner magnetosphere $[10,13]$ as well as in related laboratory experiments [15]. Observed wave amplitudes are strong enough to provide electron trapping and acceleration in the inhomogeneous dipolar magnetic field of the Earth [50, 59]. Third, spacecraft recently detected very intense bursts of parallel electric fields in the equatorial inner magnetosphere [60-62], magnetophause [63, 64], and magnetotail $[65,66]$ regions. These bursts represent electrostatic solitary waves [44] and can effectively trap and scatter electrons up to relativistic energies [67-69]. Other possible applications correspond to strong Langmuir waves observed in solar wind [70,71] and intense kinetic Alfven waves predicted in solar corona [72] and solar wind [73].

Finally, inertial confinement fusion experiments at the National Ignition Facility (NIF) involving intense laserplasma interaction have demonstrated the presence of strong levels of reflectivity due to backward stimulated Raman scattering [74], driving forward-propagating high amplitude Langmuir waves [17]. Such waves can in turn produce a significant population of suprathermal electrons that may modify Raman scattering but also penetrate inside the capsule and preheat the fuel, reducing its compression and compromising ignition [18, 19]. Accurately determining this high-energy electron tail is therefore crucial for both direct and indirect drive laser fusion, and our proposed approach could allow a fast exploration of this effect over a wide range of parameters without resorting to time-consuming particle simulations or experiments.

Figure 10 demonstrates an interesting effect of nonlinear wave-particle interactions: both the growth and decrease of average energy of a charged particle ensemble depend on the initial distribution function and wave characteristics. If the majority of the resonant particles can be trapped by the waves, then the average energy increases significantly. In the opposite situation when the majority of particles can be scattered without trapping, the average energy decreases. Therefore, there are some initial distributions for which the average energy does not change, whereas for other distributions, particle trapping generates a high-energy tail population. In the presence of permanent particle sources and losses, such a system can be balanced and provide acceleration of a small particle population at the cost of slightly energy decrease of a much larger particle population.

There are two important conditions (assumptions) which should be satisfied. First of all, particle motion should be sufficiently stochastic, to exclude correlations between phase values $\phi$ which a particle has at two successive resonant interactions. Second, the variable $\theta$ in Eq. (14) should be distributed uniformly within the range $\theta \in[0,1]$. The first condition is satisfied due to magnetic field inhomogeneity $b(z)$, which leads to a dependence of the particle bounce period given by Eq. (21) on energy. In this case, a small change of energy in the resonance results in a change of bounce period $\delta T$ and, thus, a phase change $\delta \phi \sim \omega \delta T$ between two resonant interactions. This removes from the system any possible correlation between resonant phase values (see, e.g., corresponding estimates in $[75,76])$. This effect of resonant particle chaotic motion in inhomogeneous magnetic field has been checked in [40, 77-79]. The satisfaction of the second condition was checked numerically in [80]. Moreover, Fig. 7 demonstrates that Eq. (14) is accurate enough.

We consider a simplified system with the harmonic wave $\sim \sin \phi$ and stationary background magnetic field. For more realistic situations, both wave amplitude modulation (i.e., propagation of localized wave packets) and external noise of magnetic field can be present in the system. Effect of wave modulation reduces the probability of trapping [81, 82] and can be included into Eq. (20) through the multiplication factors for $V_{h}$. High-frequency nonresonant fluctuations of magnetic field (magnetic field noise) result in destruction of the invariant $\int K d \phi$ of trapped particle motion. This effect reduces the time which particles spend in trapping region $[83,84]$. The corresponding reduction of $\Delta h$ (i.e., a modification of the function $\left.h_{*}(h)\right)$ can be evaluated using approach proposed in $[84,85]$.

Equation (20) contains terms of the order of $\sim \varepsilon$ (diffusion coefficient) and $\sim \sqrt{\varepsilon}$ (drift velocity). Because $\varepsilon \ll 1$, terms $\sim \sqrt{\varepsilon}$ dominate the $f(h)$ evolution and mask effects of slow diffusion $\sim \varepsilon$. For a small wave amplitude, $a<1$, only the term $\sim \varepsilon$ is left and the $f(h)$ evolution becomes much slower. As a result, Eq. (20) mainly pretends to describe the $f(h)$ evolution with an accuracy $\sim \sqrt{\varepsilon}$. To increase the level of accuracy, one should calculate the second order correction to $V_{h}$ (i.e., a term $\sim \varepsilon$ ).

The nonlocal term $d V_{h} / d h\left(f-f_{*}\right) \Theta(h)$ in Eq. (20) can be written in the presented form only in systems where particle transport due to trapping is defined by a singlevalued mapping $h_{*}(h)$, i.e. when for each $h^{*}$ there exists only one $h$ solution of the equation $h+\Delta h(h)=h_{*}$. In the more general case, when transport to $h_{*}$ is possible for different $h$, the nonlocal term should be written in the form of an integral.

Equation (20) is written for a simple system comprising 
only one intense wave. Averaging over a wave ensemble requires that all waves be well separated in wave phase velocity space to exclude overlapping of resonances (otherwise, nonlinear effects stop working and quasi-linear theory should be applied $[86,87])$. For a system with intense wave ensemble, one needs to average the diffusion coefficient $D_{h h}$ and drift velocity $V_{h}$ over wave characteristics. However, direct averaging cannot be applied for the nonlocal term $d V_{h} / d h\left(f-f_{*}\right) \Theta(h)$, because for each wave the mapping $h\left(h_{*}\right)$ should be defined separately. Instead of such an averaging, one should rather consider the sum of nonlocal terms calculated for different waves of the ensemble and take them into account with weighting factors corresponding to the relative occurrence of each wave.

We have considered here a plasma system with Landau wave-particle resonance, such that the change of adiabatic invariant $\Delta I$ directly corresponds to an energy change $\Delta h$. In the more general case of cyclotron resonance, $I$ depends on both energy $h$ and particle pitchangle $\alpha$. Thus, the change of $I$ should be written as $\Delta I=(\partial I / \partial h) \Delta h+(\partial I / \partial \alpha) \Delta \alpha$. Using this equation and the conservation of energy $\tilde{H}$ from Eq. (2), one can obtain both $\Delta h$ and $\Delta \alpha$. The corresponding kinetic equation (18) will be two-dimensional with $f=f(h, \alpha)$, i.e. particle trapping and particle drift will transport particles in a $2 \mathrm{D}$ space. The corresponding expressions for trapping probabilities and drift velocity in a system with cyclotron resonances have already been derived in $[22,51,89]$.

To conclude, we have described the nonlinear waveparticle interaction using appropriate models of particle trapping and nonlinear scattering. To provide equations describing the probability of trapping and drift velocity of particles, we use an analysis of particle trajectories expanded around wave-particle resonance. We have used the derived expressions for probability, drift velocity, and diffusion coefficient to construct a generalized FokkerPlanck equation including the effects of fast transport in phase space. Solutions of this equation have been validated by results of test particle numerical simulations. This new approach for the description of a large particle ensemble in a system with intense waves can be applied to many space and laboratory plasma systems.

\section{ACKNOWLEDGMENTS}

The work of A.V.A., A.A.V., and A.I.N was supported by the Russian Scientific Fund, project \# 14-12-00824.
[1] R. M. Thorne, B. Ni, X. Tao, R. B. Horne, and N. P. Meredith, Nature (London) 467, 943 (2010).

[2] R. M. Thorne, W. Li, B. Ni, Q. Ma, J. Bortnik, L. Chen, D. N. Baker, H. E. Spence, G. D. Reeves, M. G. Henderson, C. A. Kletzing, W. S. Kurth, G. B. Hospodarsky, J. B. Blake, J. F. Fennell, S. G. Claudepierre, and S. G. Kanekal, Nature (London) 504, 411 (2013).

[3] R. B. Horne, S. A. Glauert, N. P. Meredith, D. Boscher, V. Maget, D. Heynderickx, and D. Pitchford, Space Weather 11, 169 (2013).

[4] A. A. Vedenov, E. Velikhov, and R. Sagdeev, Nuclear Fusion Suppl. 2, 465475 (1962).

[5] W. E. Drummond and D. Pines, Nuclear Fusion Suppl. 3, 1049 (1962).

6] A. A. Vedenov, Soviet Physics Doklady 7, 1008 (1963).

[7] C. F. Kennel and F. Engelmann, Physics of Fluids 9, 2377 (1966).

[8] D. D. Ryutov, Soviet Journal of Experimental and Theoretical Physics 30, 131 (1969).

[9] L. R. Lyons, R. M. Thorne, and C. F. Kennel, J. Geophys. Res. 77, 3455 (1972).

[10] C. Cattell, J. R. Wygant, K. Goetz, K. Kersten, P. J. Kellogg, T. von Rosenvinge, S. D. Bale, I. Roth, M. Temerin, M. K. Hudson, R. A. Mewaldt, M. Wiedenbeck, M. Maksimovic, R. Ergun, M. Acuna, and C. T. Russell, Geophys. Res. Lett. 35, L01105 (2008).

[11] C. M. Cully, J. W. Bonnell, and R. E. Ergun, Geophys. Res. Lett. 35, L17S16 (2008).

[12] L. B. Wilson, III, C. A. Cattell, P. J. Kellogg, J. R. Wygant, K. Goetz, A. Breneman, and K. Kersten, Geophys. Res. Lett. 38, L17107 (2011).

[13] O. Agapitov, A. Artemyev, D. Mourenas, V. Krasnosel- skikh, J. Bonnell, O. Le Contel, C. M. Cully, and V. Angelopoulos, J. Geophys. Res. 119, 16061626 (2014).

[14] J. C. Foster, P. J. Erickson, D. N. Baker, S. G. Claudepierre, C. A. Kletzing, W. Kurth, G. D. Reeves, S. A. Thaller, H. E. Spence, Y. Y. Shprits, and J. R. Wygant, Geophys. Res. Lett. 41, 20 (2014).

[15] B. Van Compernolle, X. An, J. Bortnik, R. M. Thorne, P. Pribyl, and W. Gekelman, Physical Review Letters 114, 245002 (2015).

[16] E. M. Tejero, C. Crabtree, D. D. Blackwell, W. E. Amatucci, M. Mithaiwala, G. Ganguli, and L. Rudakov, Scientific Reports 5, 17852 (2015).

[17] D. Bénisti, O. Morice, L. Gremillet, A. Friou, and E. Lefebvre, Physics of Plasmas 19, 056301 (2012).

[18] P. Michel, L. Divol, E. L. Dewald, J. L. Milovich, M. Hohenberger, O. S. Jones, L. B. Hopkins, R. L. Berger, W. L. Kruer, and J. D. Moody, Physical Review Letters 115, 055003 (2015).

[19] E. L. Dewald, F. Hartemann, P. Michel, J. Milovich, M. Hohenberger, A. Pak, O. L. Landen, L. Divol, H. F. Robey, O. A. Hurricane, T. Döppner, F. Albert, B. Bachmann, N. B. Meezan, A. J. MacKinnon, D. Callahan, and M. J. Edwards, Physical Review Letters 116, 075003 (2016).

[20] Y. Omura, H. Matsumoto, D. Nunn, and M. J. Rycroft, Journal of Atmospheric and Terrestrial Physics 53, 351 (1991).

[21] V. Y. Trakhtengerts and M. J. Rycroft, Whistler and Alfvén Mode Cyclotron Masers in Space, by V. Y. Trakhtengerts and $M$. J. Rycroft. ISBN 978-0-521-871983. Published by Cambridge University Press, Cambridge, UK, 2008. (Cambridge University Press, 2008). 
[22] D. Shklyar and H. Matsumoto, Surveys in Geophysics 30, 55 (2009).

[23] K. B. Dysthe, J. Geophys. Res. 76, 6915 (1971).

[24] D. Nunn, Journal of Plasma Physics 6, 291 (1971).

[25] V. I. Karpman and D. R. Shklyar, Sov. JETP 35, 500 (1972).

[26] P. J. Palmadesso, Physics of Fluids 15, 2006 (1972).

[27] A. Osmane and A. M. Hamza, Physics of Plasmas 19, 030702 (2012), arXiv:1104.4760 [physics.plasm-ph].

[28] A. Osmane and A. M. Hamza, Physical Review E 85, 056410 (2012).

[29] V. V. Solovev and D. R. Shkliar, Sov. Phys. JETP 63, 272 (1986).

[30] J. M. Albert, Physics of Fluids B 5, 2744 (1993).

[31] V. Y. Trakhtengerts, J. Geophys. Res. 100, 17205 (1995).

[32] Y. Omura, N. Furuya, and D. Summers, J. Geophys. Res. 112, A06236 (2007).

[33] A. G. Demekhov, V. Y. Trakhtengerts, M. Rycroft, and D. Nunn, Geomagnetism and Aeronomy 49, 24 (2009).

[34] J. M. Albert, X. Tao, and J. Bortnik, in Dynamics of the Earth's Radiation Belts and Inner Magnetosphere, American Geophysical Union, edited by D. Summers, I. U. Mann, D. N. Baker, and M. Schulz (2013).

[35] A. V. Artemyev, A. A. Vasiliev, D. Mourenas, O. V. Agapitov, and V. V. Krasnoselskikh, Physics of Plasmas 21, 102903 (2014).

[36] D. Nunn and Y. Omura, J. Geophys. Res. 120, 2890 (2015).

[37] Y. Omura, M. Hikishima, Y. Katoh, D. Summers, and S. Yagitani, J. Geophys. Res. 114, A07217 (2009).

[38] A. G. Demekhov, Radiophysics and Quantum Electronics 53, 609 (2011).

[39] Y. Omura, D. Nunn, and D. Summers, in Dynamics of the Earth's Radiation Belts and Inner Magnetosphere, American Geophysical Union, edited by D. Summers, I. U. Mann, D. N. Baker, and M. Schulz (2013) pp. 243-254.

[40] D. R. Shklyar, Sov. Phys. JETP 53, 1197 (1981).

[41] A. V. Artemyev, A. A. Vasiliev, D. Mourenas, O. Agapitov, V. Krasnoselskikh, D. Boscher, and G. Rolland, Geophys. Res. Lett. 41, 57275733 (2014).

[42] Y. Omura, Y. Miyashita, M. Yoshikawa, D. Summers, M. Hikishima, Y. Ebihara, and Y. Kubota, J. Geophys. Res. 120, 9545 (2015).

[43] D. R. Shklyar, Annales Geophysicae 29, 1179 (2011).

[44] I. Y. Vasko, O. V. Agapitov, F. S. Mozer, A. V. Artemyev, and J. F. Drake, Physics of Plasmas 23, 052306 (2016).

[45] A. V. Artemyev, A. I. Neishtadt, A. A. Vasiliev, and D. Mourenas, Physics of Plasmas 23, 090701 (2016).

[46] A. Neishtadt, Journal of Applied Mathematics and Mechanics 39, 594 (1975).

[47] V. I. Arnold, V. V. Kozlov, and A. I. Neishtadt, Mathematical Aspects of Classical and Celestial Mechanics, 3rd ed., Dynamical Systems III. Encyclopedia of Mathematical Sciences (Springer-Verlag, New York, 2006).

[48] A. I. Neishtadt and A. A. Vasiliev, Nuclear Instruments and Methods in Physics Research A 561, 158 (2006), arXiv:nlin/0511050.

[49] A. I. Neishtadt, Russian Mathematical Surveys 69, 771 (2014).

[50] A. Artemyev, O. Agapitov, D. Mourenas, V. Krasnoselskikh, V. Shastun, and F. Mozer, Space Science Reviews
200, 261 (2016)

[51] A. V. Artemyev, A. A. Vasiliev, D. Mourenas, A. I. Neishtadt, O. V. Agapitov, and V. Krasnoselskikh, Physics of Plasmas 22, 112903 (2015).

[52] J. R. Cary and A. J. Brizard, Reviews of Modern Physics 81, 693 (2009).

[53] L. D. Landau and E. M. Lifshitz, Vol. 1: Mechanics, Course of Theoretical Physics (Oxford: Pergamon, 1988).

[54] A. I. Neishtadt, NATO ASI Series C. Dordrecht: Kluwer Acad. Publ. 533, 193 (1999).

[55] D. Dolgopyat, in Memoires De La Societe Mathematique De France, Memoires De La Societe Mathematique De France, Vol. 128 (2012).

[56] J. R. Wygant, A. Keiling, C. A. Cattell, R. L. Lysak, M. Temerin, F. S. Mozer, C. A. Kletzing, J. D. Scudder, V. Streltsov, W. Lotko, and C. T. Russell, J. Geophys. Res. 107, 1201 (2002).

[57] C. E. J. Watt and R. Rankin, Physical Review Letters 102, 045002 (2009).

[58] A. V. Artemyev, R. Rankin, and M. Blanco, J. Geophys. Res. 120, 10 (2015).

[59] A. Osmane and A. M. Hamza, Nonlinear Processes in Geophysics 21, 115 (2014), arXiv:1402.1691 [physics.space-ph].

[60] F. S. Mozer, S. D. Bale, J. W. Bonnell, C. C. Chaston, I. Roth, and J. Wygant, Physical Review Letters 111, 235002 (2013).

[61] D. M. Malaspina, J. R. Wygant, R. E. Ergun, G. D. Reeves, R. M. Skoug, and B. A. Larsen, J. Geophys. Res. 120, n/a (2015), 2015JA021137.

[62] F. S. Mozer, O. Agapitov, A. Artemyev, J. F. Drake, V. Krasnoselskikh, S. Lejosne, and I. Vasko, Geophys. Res. Lett. 42, 36273638 (2015).

[63] F. S. Mozer, O. A. Agapitov, A. Artemyev, J. L. Burch, R. E. Ergun, B. L. Giles, D. Mourenas, R. B. Torbert, T. D. Phan, and I. Vasko, Physical Review Letters 116, 145101 (2016).

[64] R. E. Ergun, K. A. Goodrich, F. D. Wilder, J. C. Holmes, J. E. Stawarz, S. Eriksson, A. P. Sturner, D. M. Malaspina, M. E. Usanova, R. B. Torbert, P.-A. Lindqvist, Y. Khotyaintsev, J. L. Burch, R. J. Strangeway, C. T. Russell, C. J. Pollock, B. L. Giles, M. Hesse, L. J. Chen, G. Lapenta, M. V. Goldman, D. L. Newman, S. J. Schwartz, J. P. Eastwood, T. D. Phan, F. S. Mozer, J. Drake, M. A. Shay, P. A. Cassak, R. Nakamura, and G. Marklund, Physical Review Letters 116, 235102 (2016).

[65] Y. V. Khotyaintsev, A. Vaivads, M. André, M. Fujimoto, A. Retinò, and C. J. Owen, Physical Review Letters 105, 165002 (2010).

[66] R. E. Ergun, K. A. Goodrich, J. E. Stawarz, L. Andersson, and V. Angelopoulos, J. Geophys. Res. 120, 1832 (2015).

[67] A. V. Artemyev, O. Agapitov, F. Mozer, and V. Krasnoselskikh, Geophys. Res. Lett. 41, 57345739 (2014).

[68] F. S. Mozer, A. Artemyev, O. V. Agapitov, D. Mourenas, and I. Vasko, Geophys. Res. Lett. 43, 508 (2016).

[69] A. Osmane, L. B. Wilson, III, L. Blum, and T. I. Pulkkinen, Astrophys. J. 816, 51 (2016).

[70] R. E. Ergun, D. M. Malaspina, I. H. Cairns, M. V. Goldman, D. L. Newman, P. A. Robinson, S. Eriksson, J. L. Bougeret, C. Briand, S. D. Bale, C. A. Cattell, P. J. Kellogg, and M. L. Kaiser, Physical Review Letters 101, 051101 (2008). 
[71] C. Krafft, A. S. Volokitin, and V. V. Krasnoselskikh, Astrophys. J. 809, 176 (2015).

[72] Y. M. Voitenko, Solar Physics 182, 411 (1998).

[73] J. S. Zhao, Y. Voitenko, D. J. Wu, and J. De Keyser, Astrophys. J. 785, 139 (2014).

[74] J. Lindl, O. Landen, J. Edwards, and E. Moses, Physics of Plasmas 21, 020501 (2014).

[75] A. I. Neishtadt, A. V. Artemyev, L. M. Zelenyi, and D. L. Vainshtein, JETP Letters 89, 441 (2009).

[76] A. Neishtadt, A. Vasiliev, and A. Artemyev, Moscow Mathematical Journal 11, 531 (2011).

[77] V. Y. Trakhtengerts, Y. Hobara, A. G. Demekhov, and M. Hayakawa, Physics of Plasmas 6, 692 (1999).

[78] A. G. Demekhov, V. Y. Trakhtengerts, Y. Hobara, and M. Hayakawa, Physics of Plasmas 7, 5153 (2000).

[79] J. M. Albert, J. Geophys. Res. 115, A00F05 (2010).

[80] A. P. Itin, A. I. Neishtadt, and A. A. Vasiliev, Physica D: Nonlinear Phenomena 141, 281 (2000).

[81] X. Tao, J. Bortnik, R. M. Thorne, J. M. Albert, and
W. Li, Geophys. Res. Lett. 39, L06102 (2012).

[82] A. Artemyev, V. Krasnoselskikh, O. Agapitov, D. Mourenas, and G. Rolland, Physics of Plasmas 19, 122901 (2012).

[83] D. Nunn, Plan. Sp. Sci. 34, 429 (1986).

[84] A. V. Artemyev, D. Mourenas, O. V. Agapitov, D. L. Vainchtein, F. S. Mozer, and V. V. Krasnoselskikh, Physics of Plasmas 22, 082901 (2015).

[85] A. Artemyev, D. Vainchtein, A. Neishtadt, and L. Zelenyi, Phys. Rev. E 84, 046213 (2011).

[86] V. I. Karpman, Space Science Review 16, 361 (1974).

[87] V. D. Shapiro and R. Z. Sagdeev, Physics Reports 283, 49 (1997)

[88] D. Mourenas, A.V. Artemyev, O.V. Agapitov, V. Krasnoselskikh, and F.S. Mozer, J. Geophys. Res. 120, 3665 (2015).

[89] J. M. Albert, J. Geophys. Res. 105, 21191 (2000). 